> Les observations biomédicales ne deviennent une source de connaissance qu'après un débat entre chercheurs. Au cours de ce débat, la citation des études antérieures tient un rôle majeur, mais les travaux académiques qui en évaluent l'usage sont rares. Ils ont cependant pu révéler deux types de problèmes: les biais de citation et les écarts de sens entre l'étude antérieure citée et ce qu'en dit l'article citant. Dans cette revue, nous synthétisons ces travaux et en dégageons les principales caractéristiques: les études favorables à la conclusion des auteurs citants sont plus souvent citées que celles qui les questionnent; des écarts de sens majeurs affectent environ $10 \%$ des citations. Nous illustrons par deux exemples les conséquences de ce mésusage des citations. <

\section{Le mésusage \\ des citations et \\ ses conséquences \\ en médecine}

Estelle Dumas-Mallet, Thomas Boraud,

François Gonon

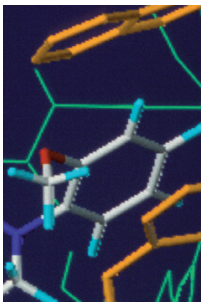

Institut des maladies neurodégéneratives et CNRS UMR 5293, Université de Bordeaux, 146 rue Léo Saignat, 33076 Bordeaux, France. francois.gonon@u-bordeaux.fr

le manque de réplicabilité de certaines observations et données expérimentales (i.e., la difficulté pour des chercheurs à confirmer, à l'aide d'autres observations, un résultat publié antérieurement) [1, 2]. Par contre, I'utilisation des citations dans la littérature scientifique n'a été que rarement évaluée. Quelques études datant des années 1970 (reprises dans [3]) portaient sur les erreurs de référence (e.g., date de publication, pages du journal), mais ce problème a quasiment disparu grâce aux bases de données et aux outils informatiques désormais disponibles. Reste ce qui est essentiel à la qualité du débat scientifique: I'usage judicieux des citations d'études antérieures. Quelques études de cas ont montré que le mésusage des citations peut générer, pendant des années, des dogmes scientifiques qui ne sont pas fondés. Parmi celles-ci, la plus connue est celle de Steven Greenberg parue en 2009 (qui affiche 240 citations dans SCOPUS, en mars 2021) [4]. Celui-ci a analysé le réseau d'études et de citations relatives à l'hypothèse selon laquelle la protéine $\beta$-amyloïde, qui joue un rôle majeur dans la maladie d'Alzheimer, pourrait être aussi produite au niveau des muscles squelettiques, et donc être impliquée dans des myosites à inclusion. Greenberg a montré que les travaux qui réfutent cette hypothèse sont beaucoup moins cités que ceux qui la soutiennent, révélant ainsi un biais de citation. Il a également observé plusieurs types de distorsion des citations où le message délivré par l'étude antérieure citée différait en fait de ce qu'en disaient les auteurs citant cette référence [4].

Nous présentons ici une synthèse de la littérature académique décrivant et quantifiant les différentes formes

Vignette (Photo ๑ Inserm/Wermuth, Camille-Georges) 
de mésusage des citations scientifiques. Nous détaillons ensuite deux exemples où des citations biaisées et inappropriées ont joué un rôle dans deux crises sanitaires majeures : la crise des opioïdes au début des années 2000 aux États-Unis, et la controverse médiatique en faveur de l'hydroxychloroquine (HCQ) en 2020 en France.

\section{Objectifs et méthodes}

De l'étude de Steven Greenberg [4] se dégagent deux grandes formes de mésusage des citations : les biais de citations et les distorsions de citations. À part cette étude, les travaux consacrés au mésusage des citations ont abordé séparément, soit les biais de citations, soit les distorsions de citations. Nous les présenterons donc dans deux sections distinctes, même si, comme l'a montré Greenberg [4], ces deux formes de mésusage peuvent se combiner dans une même publication. La présente analyse s'appuie sur trois revues systématiques contenant des méta-analyses: I'une consacrée aux biais de citations [5] et les deux autres aux distorsions des citations $[6,7]$. Ces trois revues ont été identifiées par une recherche dans la base de données PubMed du National Center for Biotechnology Information (NCBI), avec les mots clés « citation bias » ou « quotation accuracy » recherchés dans le titre ou le résumé d'articles définis comme « review » ou «systematic review ». Pour rendre compte de la littérature la plus récente, nous avons recherché les études postérieures à ces trois revues en examinant, via SCOPUS, tous les articles les citant.

\section{Les biais de citation}

On peut distinguer deux biais de citation (Tableau I). Le premier est une caractéristique générale des études citées : celles qui rapportent un effet positif sont plus souvent citées que celles favorables à l'hypothèse nulle (i.e. une absence d'effet). Le deuxième dépend de l'article citant la référence : il consiste, pour les auteurs, à citer préférentiellement les travaux antérieurs en accord avec leur conclusion. Ces deux biais sont souvent combinés. Par exemple, Athina Tatsioni et al. ont étudié l'ensemble des publications citant deux études observationnelles publiées en 1993 et affirmant qu'une diète enrichie en vitamine $\varepsilon$ diminuait le risque de maladies cardiovasculaires [9]. Fin 2006, ces deux études initiales avaient été respectivement citées 1395 et 1234 fois [9]. En 2000, une large étude randomisée et contrôlée avait pourtant montré que la vitamine $\varepsilon$ n'apportait aucun bénéfice [9]. D'autres études randomisées et une méta-analyse publiée en 2004 confirmèrent cette absence d'effet protecteur. Pourtant, les deux études initiales étaient encore largement citées en 2005, et la moitié des articles où apparaissaient ces citations restaient favorables à la vitamine $\varepsilon$, en particulier ceux qui omettaient de citer les études randomisées [9]. Tatsioni et al. ont observé le même usage préférentiel de citations favorables à des études initiales, qui ont été ensuite contredites par des études plus solides, dans deux autres domaines: l'effet supposé protecteur du $\beta$-carotène contre le cancer, et celui des estrogènes dans la maladie d'Alzheimer [9]. Comme celle de Greenberg [4], l'étude de Tatsioni et al. [9] illustre le fait que les biais de cita- tion génèrent des dogmes non fondés et ralentissent ainsi le progrès des connaissances.

Les études évaluant les biais de citation dans la littérature biomédicale ont fait l'objet d'une seule revue systématique publiée par Bram Duyx et al. en 2017 [5]. $\varepsilon n$ effet, selon ces auteurs, leur revue est la première du genre. Elle a identifié 38 études et a pu en compiler 24 par des méta-analyses. Cette analyse a confirmé que les études rapportant un effet statistiquement significatif sont plus souvent citées que celles défendant l'hypothèse nulle $(O R=1,6 ; I C 1,3-1,8)[5]$. De plus, les études favorables à la conclusion des auteurs sont plus souvent citées que celles qui la questionnent $(O R=2,7$; IC 2,0$3,7)[5]$. Pour rendre compte des études postérieures à cette revue systématique, nous avons examiné tous les articles la citant, soit 8 revues et 19 études distinctes dans le domaine biomédical. Ces huit articles de revue couvrent d'autres questions que les biais de citation. La revue systématique de Bram Duyx et al. [5] semble donc actuellement la seule disponible sur le sujet. Parmi les 19 études originales citant Duyx et al., nous en avons identifiées 5 décrivant des biais de citations pour différentes questions d'épidémiologie (e.g., l'association entre la pollution par le moteur Diesel et le cancer du poumon). Ces cinq études ont été réalisées par le même groupe et ont été reprises dans une sixième publication analysant les réseaux de citations correspondants [10]. Cette analyse secondaire confirme que les biais de citation favorisant les résultats en accord avec l'hypothèse des auteurs sont fréquents en épidémiologie [10]. Enfin, deux études dans le domaine du diagnostic en radiologie ont montré que les travaux présentés comme positifs par leurs auteurs sont plus souvent cités. Ce biais de citation pourrait ainsi conduire à surestimer l'efficacité des tests radiologiques $[11,12]$.

\section{Les distorsions de citation}

Les distorsions de citation sont des écarts de sens entre le message délivré par l'étude antérieure citée et ce qu'en disent les auteurs citant cette référence. La mise en évidence de ces écarts est une entreprise difficile et chronophage puisqu'elle exige la lecture attentive, par un chercheur compétent dans le domaine, de l'article citant et de tous les articles cités en référence. Cela explique que peu d'études académiques se soient intéressées à ces distorsions de citations. L'étude quantitative la plus ancienne est très probablement celle de Gerald de Lacey et al. publiée en 1985 dans le British Medical Journal, puisque, selon ces auteurs, aucune étude antérieure n'avait abordé cette question [3]. Ces auteurs ont étudié les articles parus dans le premier numéro de l'année 


\section{A. Biais de citation selon Duyx et al. [5] :}

1) Citation préférentielle des études positives au détriment de celles défendant l'hypothèse nulle.

2) Citation préférentielle des études en accord avec l'hypothèse des auteurs.

\section{B. Distorsion des citations :}

1) Classification évaluative selon Jargas et Baethge [6], et Mogull [7]:

- distorsions mineures (i.e., sans influence sur le sens général de la citation)

- distorsions majeures (i.e., important écart de sens entre l'étude citée et ce qu'en disent les auteurs la citant).

2) Classification descriptive selon Pavlovic et al. [8] :

- citation de résultats inexistants

- interprétation inexacte des résultats cités

- données quantitatives attribuées à une étude citée et rapportées de manière inexacte

- citation dans un contexte inapproprié

- attribution erronée (i.e., rapporter des résultats comme provenant d'une publication citée alors qu'ils ont été rapportés dans une autre publication)

- méthodes citées de manière inexacte

Tableau I. Classification des différentes formes de mésusage des citations.

1984 de six journaux biomédicaux : British Medical Journal, The Lancet, The New England Journal of Medicine, Clinical Radiology, British Journal of Surgery et British Journal of Hospital Medicine. Pour chacun de ces six numéros, les auteurs ont sélectionné au hasard 50 citations et ont recherché un écart de sens entre l'article citant et l'étude originale citée. Selon les journaux, ils ont détecté entre 10 et $20 \%$ de citations déformées avec une moyenne de $15 \%$ pour l'ensemble des six numéros, sans différence notable entre les trois journaux prestigieux (British Medical Journal, The Lancet, The New England Journal of Medicine) et les trois autres. Les auteurs ont classifié ces distorsions en distorsions triviales $(2,7 \%)$, légèrement trompeuses ( $6 \%$ ) et sérieusement trompeuses $(6,3 \%)$. Pour ces dernières, ils donnent un exemple concernant le traitement des abcès hépatiques. L'article affirme, citation à l'appui, que les patients traités avec un antibiotique et un drainage chirurgical présentent une mortalité de $8 \%$. En fait, l'étude antérieure citée précise que la mortalité de $8 \%$ a été observée dans une sous-population de patients présentant un seul abcès, mais que, pour les patients avec de multiples abcès, la mortalité après traitement est de $71 \%$.

Deux revues systématiques récentes ont compilé l'ensemble des travaux académiques portant sur la distorsion des citations. La première a identifié 28 études couvrant le domaine médical au sens large (i.e. incluant le paramédical) et dont certaines correspondaient à des résumés accompagnant des présentations dans des congrès [6]. La deuxième, plus récente mais plus restrictive, n'a inclus que 15 études examinées par un comité de lecture et couvrant strictement le domaine médical [7]. Ces 15 études étaient déjà incluses dans la première revue. Comme de Lacey et al. [3], ces deux revues systématiques ont qualifié ces distorsions, soit de mineures (i.e. sans conséquence sérieuse pour le lecteur), soit de majeures (i.e. sérieusement trompeuses) (Tableau l). Le pourcentage de citations sérieusement trompeuses, estimé par Hannah Jergas et Christopher Baethge [6], était de 11,9\% (avec un intervalle de confiance [IC] à $95 \%$ compris entre 8,4 et 16,6\%). Selon Scott Mogull [7], le pourcentage global de distorsions serait de $14,5 \%$ (avec un IC à $95 \%$ compris entre 10,5 et $18,6 \%)$. Parmi elles, les deux tiers représenteraient des distorsions majeures (64,8\% avec un IC à $95 \%$ compris entre 56,1 et $73,5 \%$ ). Ces deux revues systématiques s'accordent donc pour estimer à environ $10 \%$ le pourcentage de citations sérieusement trompeuses $[6,7]$.

Pour identifier des études postérieures à ces deux revues systématiques, nous avons examiné toutes les publications les citant, soit 26 articles distincts. Seuls cinq d'entre eux apportent de nouvelles observations concernant les distorsions de citation en biomédecine $[8,13-16]$. Le premier article a analysé 300 citations extraites de huit journaux spécialisés en chirurgie et a détecté $6 \%$ de distorsions majeures [13]. Dans le second article, ce pourcentage atteignait $22 \%$ pour 235 citations faisant référence à 51 articles parus dans des revues de psychiatrie en langue allemande [14]. L'analyse réalisée dans le troisième article portait sur 250 citations prises au hasard dans cinq revues prestigieuses à facteurs d'impact élevés ou très élevés (Science Advances, Nature Communication, the 
Proceedings of the National Academy of Sciences U S A, Science, Nature) [15]. Selon cette analyse des articles citants, les affirmations soi-disant appuyées par 21 citations (soit 8,5\%) n'étaient en fait nullement étayées par l'article cité [15]. La quatrième étude décrit les distorsions de citations dans dix rapports officiels du gouvernement britannique concernant la santé mentale [16]. Sur les 236 affirmations présentées dans ces rapports et appuyées par une référence, 45 (soit 19\%) sont des distorsions majeures [16]. Enfin, la dernière étude est particulièrement remarquable par sa méthodologie et son ampleur [8]. Elle repose sur 27 publications concernant différents domaines de la recherche biomédicale et sélectionnées en raison du grand nombre de citations qu'elles ont reçues. Les auteurs ont examiné les 4535 articles citant ces 27 études, ce qui les a conduits à vérifier l'exactitude de 7438 citations. Parmi celles-ci, ils ont détecté 688 citations inexactes, soit un taux de 9,2\%. Ils les ont classifiées de manière descriptive (Tableau I). Les trois principales formes d'inexactitudes, représentant $70 \%$ du total, sont: la citation de données inexistantes dans l'article original $(38,4 \%)$, la mention de données quantitatives inexactes $(16,6 \%)$ et l'interprétation biaisée des observations citées $(15,4 \%)$.

Les études quantitatives explorant la distorsion des citations dans la littérature biomédicale sont peu nombreuses, mal connues et laissent inexplorés plusieurs domaines, comme la cancérologie $[6,8]$. Pourtant, toutes ces études concluent que les distorsions majeures de citation sont loin d'être rares dans cette littérature : elles affectent entre 6 et $20 \%$ des citations. Les conséquences de ces distorsions en santé publique et pour l'avancement de la recherche biomédicale sont, de plus, rarement évoquées autrement qu'en termes généraux.

Nous illustrons ici les conséquences en santé publique de ces distorsions et biais de citation avec deux exemples liés à des crises sanitaires majeures récentes.

\section{La crise des opioïdes}

Aux États-Unis, le taux de décès par overdose accidentelle d'opioïdes prescrits par un médecin a été multiplié par quatre entre 1999 et 2010 [17]. Ce taux s'est stabilisé entre 2010 et 2013, à la suite de mesures rendant plus difficile la prescription des opioïdes [17]. Par contre, entre 2010 et 2016 les taux de décès par overdose accidentelle d'héroïne et de fentanyl ${ }^{1}$ (hors prescription médicale) ont été multipliés respectivement par 5 et 9 [17]. Plusieurs études suggèrent que l'usage illégal d'héroïne et de fentanyl, à partir de 2010, a remplacé chez de nombreux patients, celui des opioïdes précédemment prescrits [17, 18]. Au total, la crise des opioïdes serait responsable de 770000 décès aux États-Unis entre 1999 et 2017 [18]. Elle expliquerait la baisse de l'espérance de vie observée depuis 2014 [19], la plus significative depuis la grande épidémie de grippe de 1918 [18].

Cette crise a été précipitée, à partir de 1996, par la promotion très agressive d'opiacés antalgiques par l'industrie pharmaceutique [20].

1 L'un des opiacés de synthèse les plus puissants, distribué par le laboratoire Janssen, division pharmaceutique de Johnson \& Johnson.
Ce discours promotionnel avançait trois arguments. Premièrement, il pointait le fait que les douleurs chroniques, hors cancer, sont mal soignées, alors qu'elles affectent durablement la qualité de vie des patients. Deuxièmement, il affirmait que les opioïdes antalgiques étaient très efficaces contre toutes les douleurs chroniques. Troisièmement, contrairement aux idées antérieures, il affirmait également que ces antalgiques n'entraîneraient que très rarement une dépendance, voire une toxicomanie. En particulier, après avoir obtenu en 1996 une autorisation de mise sur le marché pour une forme retard de l'oxycodone (0xyContin ${ }^{\mathrm{TM}}$ ), la compagnie Perdue Mundipharma a largement diffusé cet argumentaire aussi bien auprès des médecins que du grand-public [20]. Le premier constat est encore largement accepté. Par contre, l'efficacité des opiö̈des antalgiques pour soulager les patients souffrant de douleurs chroniques non cancéreuses est plus discutable et n'a été étudiée que pour des durées inférieures à six mois [21, 22]. Par rapport à un traitement placebo, cette efficacité est statistiquement significative, mais cliniquement modeste : le traitement ne montre une amélioration de la douleur que de 0,69 $\mathrm{cm}$ sur une échelle de $10 \mathrm{~cm}$ représentant visuellement le niveau de douleur [21]. De plus, la comparaison avec d'autres traitements, médicamenteux ou non, ne montre pas de supériorité des opioïdes [21].

Pour soutenir l'affirmation selon laquelle le risque de dépendance et de toxicomanie associé aux opioïdes est négligeable, une partie de l'industrie pharmaceutique ainsi que de nombreux auteurs ont cité une étude parue en 1980 dans The New England Journal of Medicine dont le titre était sans ambiguïté : Addiction Rare in Patients Treated with Narcotics $»^{2}$ [23]. Au 30 mars 2017, cette étude avait été citée par 608 articles scientifiques, dont 439 adhéraient à sa conclusion [24]. En fait, il s'agissait d'une lettre de cinq phrases, adressée à l'éditeur de la revue, mentionnant que parmi 11882 patients hospitalisés et ayant reçu au moins une fois un opioïde, les auteurs n'avaient observé que quatre cas d'addiction. Ils concluaient : «... that despite widespread use of narcotic drugs in hospitals, the development of addiction is rare in medical patients with no history of addiction $»^{3}$ [23]. Parmi les 608 articles ayant cité cette brève note, 491 omirent de mentionner qu'il s'agissait de patients hospitalisés, et que sa conclusion n'était donc pas pertinente pour le traitement des douleurs chroniques non cancéreuses en ambulatoire [24]. Selon plusieurs

\footnotetext{
2 «Les cas d'addiction sont rares chez les patients traités par des narcotiques 》. 3 «... que malgré l'usage largement répandu de narcotiques dans les hôpitaux, le développement de la dépendance est rare chez les patients traités sans antécédent de dépendance ».
} 
observateurs, la large diffusion de cette citation inappropriée a contribué au déclenchement de la crise des opioïdes [20, 24-26]. On voit donc ici un cas particulier d'une observation plus générale : le mésusage des citations est particulièrement fréquent dans les documents promotionnels publiés par l'industrie pharmaceutique [27-29]. D'autres groupes d'intérêt, comme l'industrie du tabac, en ont fait également usage [30].

\section{La promotion de l'hydroxychloroquine comme traitement de la Covid-19}

Dans le contexte de la pandémie de Covid-19, survenue au début de 2020, une équipe marseillaise publiait en avril de cette année-là, deux études réalisées sans groupe témoin, promouvant un traitement combinant l'hydroxychloroquine (HCQ) et l'azithromycine [31, 32]. Dans la première étude, portant sur 80 patients positifs dans le test $P C R^{4}$ pour le virus responsable, le SARS-CoV-2, $93 \%$ d'entre eux étaient devenus négatifs huit jours après le début du traitement [31]. Les auteurs avaient comparé cette évolution à celle rapportée par une étude chinoise dans laquelle les 191 patients examinés n'avaient pas reçu d'HCP et dont la plupart étaient encore positifs dans le test RT-PCR 20 jours après le premier test [33]. Gautret et al. affirmèrent donc, citation à l'appui, que leur traitement accélérait la disparition du virus [31]. Cette comparaison était inappropriée puisque $92 \%$ de leurs 80 patients présentaient des symptômes modérés au début du traitement et qu'un seul est décédé [31], alors que $63 \%$ des 191 patients chinois souffraient eux d'une forme sévère de la Covid-19 à leur inclusion dans l'étude, et que 54 en sont décédés [33]. L'article de Gautret et al. [31] ne mentionne pas ces caractéristiques de l'étude chinoise qu'ils citent [33]. De plus, ils citent une autre étude montrant que la sévérité des symptômes est proportionnelle à la charge virale [34] et concluent que le traitement prévient l'aggravation des symptômes, puisqu'il accélère la disparition du virus [31]. Cependant, l'article de Gautret et al. ne mentionne pas, non plus, une importante observation de cette étude citée en référence : «Les patients ayant des symptômes modérés voient leur charge virale diminuer rapidement et $90 \%$ d'entre eux deviennent négatifs au test PCR au bout de 10 jours. Par contre, les patients souffrant d'une forme sévère de l'infection sont encore positifs au bout de 10 jours » [34]. Puisque $93 \%$ des patients marseillais présentaient des symptômes modérés au début de leur traitement, on peut s'attendre à ce que leur charge virale ait diminué spontanément, et rien ne prouve que l'HCQ y soit pour quelque chose.

La deuxième étude de l'équipe marseillaise a porté sur 1061 patients positifs au test RT-PCR, dont $95 \%$ présentaient des symptômes modérés [32]. Les auteurs ont suivi l'évolution du résultat du test RT-PCR et, toujours en l'absence d'un groupe témoin, ont proposé la même comparaison inappropriée avec la population chinoise des 191 patients hospitalisés [33]. Au total, ces deux études [31, 32] ont affirmé l'efficacité d'un traitement à base d'HCQ par comparaison à deux études antérieures [33,

4 II s'agit plus exactement d'un test de RT-PCR dans lequel l'ARN viral du SARS-CoV-2, si ce dernier est présent, est transformé en ADNc (reverse transcription, RT) qui est alors amplifié par PCR (polymerase chain reaction).
34], alors même que leur présentation objective aurait dû souligner la différence de l'état clinique des patients inclus dans ces études et conduire à rejeter cette affirmation.

Ces deux études sans groupe témoin ont été publiées sur le site de la revue Travel Med Infect Dis le 4 avril et le $1^{\text {er }}$ mai 2020, mais leurs résultats ont été rendus public plusieurs jours auparavant, via les média grand-public et les réseaux sociaux. Ils ont contribué à nourrir l'espoir pour la population, mais aussi des professionnels de santé, d'un traitement sûr et efficace de la Covid-19. De fait, l'HCP a été largement prescrite dans de très nombreux pays et a été administrée à de nombreuses personnalités. Elle a également été introduite dans de nombreux essais cliniques destinés à évaluer plusieurs molécules et enrôlant des milliers de patients. Ces différents essais ont montré que I'HCQ n'était pas un traitement efficace de la Covid-19 [35-37], ce qui a éteint la controverse au cours de l'été 2020. Au début de la pandémie, cette controverse a pris une intensité considérable, alimentée par les média traditionnels et les réseaux sociaux. Plusieurs chercheurs, en France, en Suisse [38] et au Brésil [39], ont été pris à partie et menacés de mort pour avoir critiqué le traitement par l'HCQ.

On peut noter que cette distorsion majeure des citations est en quelque sorte la conséquence de précédents manquements à l'éthique médicale. $\varepsilon$ n effet, Gautret et al. avaient auparavant publié une étude comprenant un groupe témoin [40] qui avait été vivement critiquée pour sa petite taille et sa méthodologie [41]. Cependant, Gautret et al. avaient conclu que l'HCQ était un traitement efficace de la Covid-19, et qu'il n'était donc plus éthique d'inclure dans leurs études ultérieures un traitement placebo, celui-ci étant, pour eux, défavorable au patient, comparé à l'HCQ. Sur cette base, les deux études discutées plus haut [31,32] n'ayant pas de groupe témoin, leurs auteurs ont dû comparer leurs résultats à des données publiées antérieurement. En utilisant cette comparaison, ils ont omis des informations majeures qu'apportait pourtant la littérature citée, ce qui leur a permis de continuer à affirmer l'efficacité de leur traitement ${ }^{5}$. Dans ce contexte, la mise

\footnotetext{
5 Dans un récent article publié en 2021 sous forme d'une « Letter to the Editor » (Gautret et al., International Journal of Antimicrobial Agents 2021 ; 57 : 106242) intitulée «Clinical efficacy and safety profile of hydroxychloroquine and azithromycin against COVID-19 », les auteurs précisent : “...To this end, we reanalyzed our data on all patients enrolled in our study $(n=$ 42) and, in addition to viral clearance over time, we analyzed clinical outcomes, including the need for oxygen therapy, transfer to intensive care unit (ICU), death and length of stay at hospital. Requirement for oxygen therapy, transfer to ICU and death did not significantly differ between groups." Le seul point que les auteurs continuent à revendiquer est désormais : "Length of stay at hospital and viral persistence were significantly shorter in the treated patients group, compared to the control group".
} 
en garde rappelant la nécessité des essais contrôlés et randomisés, parue dans médecine/ science $[42,43](\rightarrow)$, trouve toute sa pertinence.

\section{Conclusion}

Le mésusage des citations dans la littérature biomédicale génère des dogmes non fondés et nuit au progrès des connaissances. Dans certains cas, ses conséquences se sont révélées dramatiques pour les patients. Peu étudié et trop méconnu, ce mésusage affecte tout autant la crédibilité de la recherche biomédicale que le manque de réplicabilité des résultats et des observations. Plusieurs des articles que nous citons ici proposent des recommandations pour limiter le mésusage des citations et ses conséquences $[6,8]$. Nous reprenons ici les principales. La toute première concerne les lecteurs de cette littérature biomédicale: ils devraient être mieux informés d'un possible mésusage et ne pas hésiter à vérifier, dans les articles source, la véracité des citations. Deuxièmement, les relecteurs chargés d'examiner les manuscrits avant publication dans des revues scientifiques devraient examiner soigneusement la pertinence et l'exactitude de toutes les citations des articles qu'ils expertisent. Ils devraient aussi demander aux auteurs d'ajouter des références quand la discussion de la littérature antérieure leur apparaît par trop déséquilibrée. Troisièmement, les éditeurs de revues biomédicales devraient demander à leurs relecteurs plus de vigilance quant au bon usage des citations. Ils pourraient aussi mentionner dans la note aux auteurs que leur usage des citations sera examiné de près par leurs relecteurs. Enfin, Pavlovic et al. proposent de nombreuses recommandations aux auteurs [8]. Elles sont de bon sens, mais sont impuissantes à contrecarrer la cause principale du mésusage des citations selon Jergas et al. [6] : le biais de confirmation qui nous fait retenir préférentiellement les informations en accord avec nos opinions. $\diamond$

\section{LIENS D'INTÉRÊT}

Les auteurs déclarent n'avoir aucun lien d'intérêt concernant les données publiées dans cet article.

\section{SUMMARY}

Citation misuse and its effects on public health

In order to effectively contribute to scientific knowledge, biomedical observations have to be validated and debated by scientists in the relevant field. Along this debate that mainly takes place in the scientific literature, citation of previous studies plays a major role. However, only a few academic studies have quantitatively evaluated the suitability and accuracy of scientific citations. Here we review these academic studies. Two types of misuse have been pointed out: Citation bias and citation distortion. First, scientific citations favor positive results and those supporting authors' conclusion. Second, many statements linked to a reference actually misrepresent the referenced findings. About $10 \%$ of all citations in biomedicine are strongly inaccurate and misleading for the reader. Finally, we give two examples illustrating how some citation misuses do affect public health: The opioid crisis in the USA and the unjustified fostering of hydroxychloroquine for Covid-19 treatment in France. $\diamond$

\section{RÉFÉRENCES}

1. loannidis JP. How to make more published research true. PLoS Med 2014 ; 11 : el001747.

2. Prinz F, Schlange T, Asadullah K. Believe it or not: how much can we rely on published data on potential drug targets? Nat Rev Drug Discov 2011 ; $10: 712$.

3. De Lacey G, Record C, Wade J. How accurate are quotations and references in medical journals? Br Med J 1985 ; 291 : 884-6.

4. Greenberg SA. How citation distortions create unfounded authority: analysis of a citation network. BMJ 2009 ; 339 : b2680.

5. Duyx B, Urlings MJE, Swaen GMH, et al. Scientific citations favor positive results: a systematic review and meta-analysis. J Clin Epidemiol 2017 ; $88: 92-101$.

6. Jergas $\mathrm{H}$, Baethge $\mathrm{C}$. Quotation accuracy in medical journal articles-a systematic review and meta-analysis. Peer) 2015 ; 3 : el364.

7. Mogull SA. Accuracy of cited facts in medical research articles: a review of study methodology and recalculation of quotation error rate. PLOS One 2017 ; 12 : e0184727.

8. Pavlovic V, Weissgerber T, Stanisavljevic D, et al. How accurate are citations of frequently cited papers in biomedical literature? Clin Sci (Lond) 2021 ; $135: 671-81$.

9. Tatsioni A, Bonitsis NG, loannidis JP. Persistence of contradicted claims in the literature. JAMA $2007 ; 298: 2517-26$.

10. Urlings MJE, Duyx B, Swaen GMH, et al. Citation bias and other determinants of citation in biomedical research: findings from six citation networks. J Clin Epidemiol 2021 ; 132 : 71-8.

11. Frank RA, Sharifabadi AD, Salameh JP, et al. Citation bias in imaging research: are studies with higher diagnostic accuracy estimates cited more often? Eur Radiol 2019 ; 29 : 1657-64.

12. Treanor L, Frank RA, Salameh JP, et al. Selective citation practices in imaging research: are diagnostic accuracy studies with positive titles and conclusions cited more often? Am J Roentgenol 2019; 213 : 397-403.

13. Armstrong MF, Conduff JH, 3rd, Fenton JE, Coelho DH. Reference errors in otolaryngology-head and neck surgery literature. Otolaryngol Head Neck Surg $2018 ; 159: 249-53$.

14. Baethge C. Importance, errors, and patterns of quotations to psychiatric original articles. Pharmacopsychiatry $2020 ; 53: 247-55$.

15. Smith N, Cumberledge A. Quotation errors in general science journals: quotation errors in science journals. Proc Roy Soc A 2020 ; 476 : 20200538.

16. Hui A, Rains LS, Todd A, et al. The accuracy and accessibility of cited evidence: a study examining mental health policy documents. Soc Psychiatry Psychiatr Epidemiol 2020 ; 55 : 111-21.

17. Jalal H, Buchanich JM, Roberts MS, et al. Changing dynamics of the drug overdose epidemic in the United States from 1979 through 2016. Science 2018 ; 361 : eaaul184.

18. Healton C, Pack R, Galea S. The opioid crisis, corporate responsibility, and lessons from the tobacco master settlement agreement. JAMA 2019 322 : 2071-2.

19. Woolf SH, Schoomaker $\mathrm{H}$. Life expectancy and mortality rates in the United States, 1959-2017. JAMA 2019; 322 : 1996-2016.

20. Van Zee A. The promotion and marketing of oxycontin: commercial triumph, public health tragedy. Am J Public Health 2009 ; 99 : 221-7.

21. Busse JW, Wang L, Kamaleldin M, et al. Opioids for chronic noncancer pain: a systematic review and meta-analysis. JAMA 2018 ; $320: 2448-60$.

22. Chou R, Turner JA, Devine EB, et al. The effectiveness and risks of longterm opioid therapy for chronic pain: a systematic review for a National Institutes of Health pathways to prevention workshop. Ann Intern Med $2015 ; 162: 276-86$.

23. Porter J, Jick H. Addiction rare in patients treated with narcotics. $N$ Engl J Med $1980 ; 302: 123$.

24. Leung PTM, Macdonald EM, Stanbrook MB, et al. A 1980 letter on the risk of opioid addiction. N Engl J Med 2017 ; $376: 2194-5$.

25. Becker WC, Fiellin DA. Limited evidence, faulty reasoning, and potential for a global opioid crisis. BMJ 2017 ; 358 : j3115.

26. Rummans TA, Burton MC, Dawson NL. How good intentions contributed to bad outcomes: the opioid crisis. Mayo Clin Proc 2018 ; 93 : 344-50. 


\section{RÉFÉRENCES}

27. Keng A, Coley RM. Evaluating the accuracy of citations in drug promotional brochures. Ann Pharmacother $1994 ; 28: 1231-5$.

28. Gonzalez-Santiago M, Bucher HC, Nordmann AJ. Accuracy of drug advertisements in medical journals under new law regulating the marketing of pharmaceutical products in Switzerland. BMC Med Inform Decis Mak 2008 ; $8: 61$.

29. Spielmans GI, Thielges SA, Dent AL, Greenberg RP. The accuracy of psychiatric medication advertisements in medical journals. J Nerv Ment Dis 2008 ; 196 : 267-73.

30. Bero LA, Glantz SA, Rennie D. Publication bias and public health policy on environmental tobacco smoke. JAMA $1994 ; 272: 133-6$.

31. Gautret P, Lagier JC, Parola P, et al. Clinical and microbiological effect of a combination of hydroxychloroquine and azithromycin in 80 COVID-19 patients with at least a six-day follow up: a pilot observational study. Travel Med Infect Dis 2020 : 101663

32. Million M, Lagier JC, Gautret P, et al. Early treatment of COVID-19 patients with hydroxychloroquine and azithromycin: a retrospective analysis of 1061 cases in Marseille, France. Travel Med Infect Dis 2020 : 101738.

33. Zhou F, Yu T, Du R, et al. Clinical course and risk factors for mortality of adult inpatients with COVID-19 in Wuhan, China: a retrospective cohort study. Lancet 2020 ; $395: 1054-62$.

34. Liu Y, Yan LM, Wan L, et al. Viral dynamics in mild and severe cases of COVID-19. Lancet Infect Dis $2020 ; 20: 656-7$.

35. Fiolet T, Guihur A, Rebeaud ME, et al. Effect of hydroxychloroquine with or without azithromycin on the mortality of coronavirus disease 2019 (COVID-19) patients: a systematic review and meta-analysis. Clin Microbiol Infect $2020 ; 27: 19-27$.

36. Juul S, Nielsen $\varepsilon \varepsilon$, Feinberg J, et al. Interventions for treatment of COVID-19: a living systematic review with meta-analyses and trial sequential analyses (The Living Project). PLoS Med $2020 ; 17:$ e1003293.
37. Siemieniuk RA, Bartoszko JJ, Ge L, et al. Drug treatments for covid-19: living systematic review and network meta-analysis. BMJ $2020 ; 370$ : m2980.

38. Peiffer-Smadja N, Rebeaud ME, Guihur A, et al. Hydroxychloroquine and COVID-19: a tale of populism and obscurantism. Lancet Infect Dis 2021 ; $21:$ el2l.

39. Ektorp $\varepsilon$. Death threats after a trial on chloroquine for COVID-19. Lancet Infect Dis $2020 ; 20: 661$.

40. Gautret $\mathrm{P}$, Lagier JC, Parola $\mathrm{P}$, et al. Hydroxychloroquine and azithromycin as a treatment of COVID-19: results of an open-label non-randomized clinical trial. Int J Antimicrob Agents 2020; 56 : 105949.

41. Kim AHJ, Sparks JA, Liew JW, et al. A Rush to judgment? Rapid reporting and dissemination of results and its consequences regarding the use of hydroxychloroquine for Covid-19. Ann Intern Med $2020 ; 172: 819-21$.

42. Alperovitch $A$, Lazar P. L'éthique des essais thérapeutiques. Med Sci (Paris) $2020 ; 36: 303-7$.

43. Amiel P, Chneiweiss H, Dosquet C. Covid-19 : protocoles de soins ou protocoles de recherche? Med Sci (Paris) 2020 ; $36: 521-3$.

TIRÉS À PART

F. Gonon

\section{Les Cahiers du Comité pour l'histoire de l'Inserm, $\mathrm{n}^{\circ} 2$}

\section{Du cerveau aux neurosciences: itinéraires dans la longue durée}

Le deuxième opus des Cahiers du Comité pour l'histoire de l'Inserm, « Du cerveau aux neurosciences : itinéraires dans la longue durée » vient de paraître. Prolongeant les rencontres de la journée d'étude organisée en 2020 par le Comité pour l'histoire de l'Inserm, des historiens, des chercheurs en sciences sociales, des acteurs engagés dans la recherche sur le cerveau et les neurosciences interrogent l'histoire de l'exploration du cerveau, l'évolution des technologies mises au service de la compréhension du système nerveux, les enjeux liés à la santé publique et engageant pleinement la société. Ce numéro 2, composé de 2 volumes, propose des éclairages sur la recherche sur les sciences du cerveau, l'innovation, les maladies et les patients, du Xvıl ${ }^{e}$ siècle jusqu'à nos jours. On trouve au fil de cette histoire des jalons pour comprendre le champ complexe des neurosciences contemporaines. L'iconographie, des images du cerveau, de ses représentations, des techniques d'exploration, ajoutent un éclairage supplémentaire qui est illustratif mais aussi explicatif.

Ce numéro est aussi l'occasion d'ouvrir une nouvelle rubrique consacrée aux témoignages de celles et ceux dont le métier et l'itinéraire se sont réalisés dans le monde de la recherche biomédicale et de la santé publique.

Disponible en accès libre sur iPubli.fr : https://www.ipubli.inserm.fr/handle/10608/10603

Pour toute information, contactez le secrétariat scientifique : celine.paillette@ext.inserm.fr

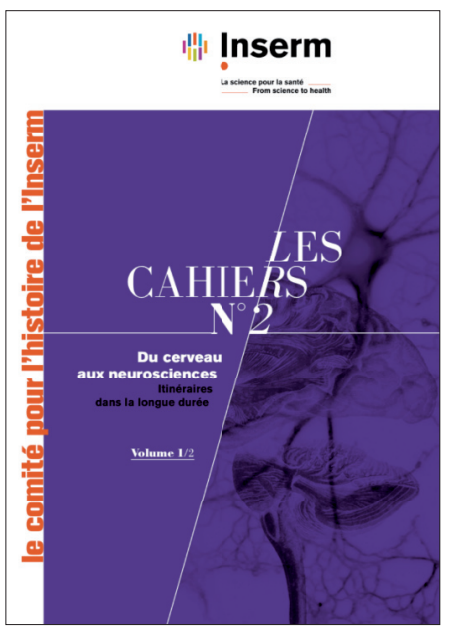

